EMMANUEL ULLMO

\title{
Sur la constante de Hida des courbes modulaires et des courbes de Shimura
}

Journal de Théorie des Nombres de Bordeaux, tome 13, $\mathrm{n}^{\circ} 1$ (2001), p. 325-337

<http://www.numdam.org/item?id=JTNB_2001_13_1_325_0>

(C) Université Bordeaux 1, 2001, tous droits réservés.

L'accès aux archives de la revue «Journal de Théorie des Nombres de Bordeaux » (http://jtnb.cedram.org/) implique l'accord avec les conditions générales d'utilisation (http://www.numdam.org/conditions). Toute utilisation commerciale ou impression systématique est constitutive d'une infraction pénale. Toute copie ou impression de ce fichier doit contenir la présente mention de copyright.

\section{Numdam}

Article numérisé dans le cadre du programme

Numérisation de documents anciens mathématiques

http://www.numdam.org/ 


\title{
Sur la constante de Hida des courbes modulaires et des courbes de Shimura
}

\author{
par EMMANUEL ULLMO
}

\begin{abstract}
RÉSUMÉ. La correspondance de Shimizu et Jacquet-Langlands donne des relations entre les quotients de la partie nouvelle de la jacobienne $J_{0}(N)$ de $X_{0}(N)$ et ceux de la partie nouvelle de la jacobienne de certaines courbes de Shimura associées. Nous comparons dans ce texte les congruences entre formes modulaires pour des quotients qui sont associés dans cette correspondance.
\end{abstract}

ABSTRACT. We compare the "modules of congruences between modular forms" of quotients of the new part of the Jacobian $J_{0}(N)$ of $X_{0}(N)$ and quotients of the new part of the Jacobian of some Shimura curves which are associated in the correspondance of Shimizu and Jacquet-Langlands.

\section{Introduction}

Soit $N$ un entier sans facteurs carrés, $X_{0}(N)$ la compactification de l'espace de module grossier des courbes elliptiques munies d'un sous groupe cyclique d'ordre $N$ et $J_{0}(N)$ la jacobienne de $X_{0}(N)$. Pour toute décomposition de $N$ sous la forme

$$
N=N^{+} N^{-},
$$

où $N^{-}$est le produit d'un nombre pair de nombres premiers, on définit la courbe de Shimura $X_{N^{+}, N^{-}}$. Cette courbe admet un modèle sur $\mathbb{Q}$ et est obtenue sur $\mathbb{C}$ comme quotient du demi-plan de Poincaré par le sous-groupe $\Gamma_{0}^{N^{+}}\left(N^{-}\right)$du groupe des unités de norme 1 dans un ordre d'Eichler de niveau $N^{+}$de l'algèbre de quaternion rationelle (indéfinie) de discriminant $N^{-}$[18] [2]. La correspondance de Shimizu et Jacquet-Langlands relie les formes nouvelles de poids deux pour $X_{0}(N)$ à celles de $X_{N^{+}, N^{-}}$qui ne sont bien définies qu'à la multiplication par une constante près.

L'étude arithmétique des courbes $X_{N^{+}, N^{-}}$est limitée par l'absence de développement de Fourier des formes différentielles due à l'absence de pointes. En particulier la théorie des congruences entre formes modulaires développée par Hida $[7,8]$ et Ribet[14, 15] ne s'étend pas dans cette situation.

Manuscrit reçu le 16 novembre 1999. 
Soit $K$ un corps de caractéristique 0 et $A$ une variété abélienne sur $K$. En reprenant la terminologie de Mazur [10], on dit qu'une variété abélienne $A^{\prime}$ définie sur $K$ est un quotient optimal de $A$ s'il existe une sous-variété abélienne $A^{\prime \prime}$ de $A$ définie sur $K$ et une suite exacte de variétés abéliennes

$$
0 \rightarrow A^{\prime \prime} \longrightarrow A \longrightarrow A^{\prime} \rightarrow 0 .
$$

On a défini dans [17] la constante de Hida d'un quotient optimal de la jacobienne d'une courbe. Rappelons brièvement cette définition et quelques propriétés.

On suppose que $J$ est la jacobienne d'une courbe $C$ projective, lisse, irréductible de genre $g \geq 1$ sur $\mathbb{C}$. On fixe un plongement $\phi$ de $C$ dans $J$, on a un isomorphisme $\phi^{*}$ entre $H^{0}\left(J, \Omega^{1}\right)$ et $H^{0}\left(C, \Omega^{1}\right)$. On notera de la même manière deux éléments de $H^{0}\left(J, \Omega^{1}\right)$ et $H^{0}\left(C, \Omega^{1}\right)$ qui se correspondent par $\phi^{*}$. Pour toute variété abélienne $A$, de dimension $a$, quotient de $J, H^{0}\left(A, \Omega^{1}\right)$ est un sous espace vectoriel de $H^{0}\left(J, \Omega^{1}\right)$. Pour toute base $\left(\alpha_{1}, \ldots, \alpha_{a}\right)$ de $H^{0}\left(A, \Omega^{1}\right)$, on pose

$$
c\left(\alpha_{1}, \ldots, \alpha_{a}\right)=\left|\frac{\operatorname{det}\left(\int_{C} \alpha_{i} \wedge \overline{\alpha_{j}}\right)}{\int_{A} \alpha_{1} \wedge \cdots \wedge \alpha_{a} \wedge \overline{\alpha_{1}} \wedge \cdots \wedge \overline{\alpha_{a}}}\right| .
$$

Soit $\zeta: J \longrightarrow A$ qui fait de $A$ un quotient de $J$. On voit facilement que $c\left(\alpha_{1}, \ldots, \alpha_{a}\right)$ est indépendant de la base $\left(\alpha_{1}, \ldots, \alpha_{a}\right)$ choisie et ne dépend que de $\zeta$. On notera $c_{\zeta}=c\left(\alpha_{1}, \ldots, \alpha_{a}\right)$. Si il n'y a pas d'ambiguïté sur la structure de quotient, on posera $c_{A}=c_{\zeta}$.

On montre que $c_{A}$ est un entier qui vaut 1 si $A=J$. On suppose maintenant que $A$ est un quotient optimal de $J$. Soit $\zeta^{\vee}: A^{\vee} \longrightarrow J$ le morphisme donné par dualité. On dispose alors d'une isogénie $\zeta \zeta^{\vee}: A^{\vee} \longrightarrow$ $A$. On note $H$ le noyau de cette isogénie, on a alors [17]

$$
c_{A}=\sqrt{|H|} .
$$

Pour un quotient optimal $A$ de la partie nouvelle $J_{0}(N)^{\text {new }}$ de la jacobienne $J_{0}(N)$ de $X_{0}(N)$ (notons que $J_{0}(N)^{n e w}$ est défini comme un quotient optimal de $\left.J_{0}(N)\right)$, la définition précédente est dû à Hida. Pour cette raison nous dirons que $c_{A}$ est la constante de Hida de $A$ et nous renvoyons aux papiers de Hida [7] [8] pour les liens entre cette constante et les congruences entre formes modulaires (voir aussi [17] pour une interprétation en termes de formes différentielles). L'avantage de la définition de Hida est qu'elle garde un sens pour tout quotient de la jacobienne d'une courbe. Elle est en particulier bien définie pour un quotient optimal de la jacobienne $J_{0}\left(N^{+}, N-\right)$.

En utilisant les résultats de Faltings (ou de Ribet dans ce cas particulier), on montre qu'un de tel quotient $A^{\prime}$ de la partie nouvelle $J_{0}\left(N^{+}, N^{-}\right)^{\text {new }}$ (encore une fois définie comme un quotient ) de $J_{0}\left(N^{+}, N^{-}\right)$est isogène à un quotient $A$ de la partie nouvelle $J_{0}(N)^{\text {new }}$ de $J_{0}(N)$. Notons que dans 
cette situation par les théorèmes de multiplicité 1 , il n'y a pas d'ambiguïté sur la structure de quotient, on parlera donc de la constante de Hida $A$ ou de $A^{\prime}$ sans référence à la structure de quotient.

Pour toute variété abélienne $A$ sur $\mathbb{Q}$ et tout nombre premier $l$, on note $\phi(A, l)$ le groupe des composantes de la fibre géométrique en $l$ du modèle de Néron de $A$. Si $\zeta: A \rightarrow B$ est un morphisme de variétés abéliennes définies sur $\mathbb{Q}$, on note $\zeta_{l *}$ le morphisme induit de $\phi(A, l)$ dans $\phi(B, l)$. On montre dans ce texte le résultat suivant :

Théorème 1.1. Soit $J_{1}=J_{0}\left(N^{+}, N^{-}\right)$. Soit $p$ et $q$ deux nombres premiers divisant $N^{-}$et $J_{2}=J_{0}\left(N^{+} p q, \frac{N^{-}}{p q}\right)$. Soient $A_{i}$ des quotients optimaux de la partie nouvelles $J_{i}^{\text {new }}$ de $J_{i}(i=1$ ou $i=2)$ qui sont isogènes. Soit $\zeta_{i}: J_{i} \rightarrow A_{i}$ les morphismes donnant la structure de quotient optimal. On a la relation

$$
c_{A_{2}}=c_{A_{1}} \frac{\left|\phi\left(A_{1}, p\right)\right|\left|\phi\left(A_{2}, q\right)\right|}{\left|\operatorname{coker} \zeta_{1, p *}\right|^{2}\left|\operatorname{Im} \zeta_{2, q *}\right|^{2}} .
$$

Dans [17] une expression pour la hauteur de Faltings d'un quotient optimal $A$ de la partie nouvelle de $J_{0}(N)$ est donné et elle fait intervenir la constante de Hida $c_{A}$.

Quand les $A_{i}$ sont des courbes elliptiques, le théorème précédent est dû à Ribet et Takahashi [16] et les méthodes de ce texte sont inspirés de leurs travaux. Donnons une application de ce théorème. On suppose que

$$
N=\prod_{i=1}^{r} p_{i} q_{i}
$$

est un produit d'un nombre pair de facteurs premiers. On pose $D_{i}=$ $\prod_{j=1}^{i} p_{j} q_{j}$ et $N_{i}=\frac{N}{D_{i}}$. On pose $J_{i}^{\prime}=J_{0}\left(N_{i}, D_{i}\right)$ et $J_{i}=J_{i}^{\prime \text { new }}$ la partie nouvelle de $J_{i}^{\prime}$. Par convention $J_{0}=J_{0}(N)^{\text {new }}$ et $J_{0}^{\prime}=J_{0}(N)$. Les variétés abéliennes $J_{i}$ sont toutes dans la même classe d'isogénie. On note

$$
\zeta_{i}: J_{i}^{\prime} \longrightarrow J_{i}
$$

l'application qui fait de $J_{i}$ un quotient optimal de $J_{i}^{\prime}$. On note $c_{0}=c_{J_{0}(N)^{\text {new }}}$ la constante de Hida de $J_{0}=J_{0}(N)^{\text {new }}$. Cette constante mesure les congruences entre l'espace des formes nouvelles et celui des formes anciennes sur $J_{0}(N)$. Quand on a remarqué que $J_{r}=J_{r}^{\prime}$ et donc que la constante de Hida de $J_{r}$ vaut 1 , une application répétée du théorème donne :

Théorème 1.2. On a la relation :

$$
c_{J_{0}(N)^{n e w}}=\prod_{i=0}^{r-1} \frac{\left|\phi\left(J_{i+1}, p_{i+1}\right)\right|\left|\phi\left(J_{i}, q_{i+1}\right)\right|}{\left|\operatorname{coker} \zeta_{i+1, p_{i+1}}\right|^{2}\left|\operatorname{Im} \zeta_{i, q_{i+1}}\right|^{2}} .
$$


On peut bien sûr permuter les $p_{i}$ et les $q_{i}$ dans les formules précédentes ou choisir une autre décomposition de $N$ en un produit de nombres premiers ; c'est une conséquence du théorème précédent que toutes les expressions que l'on peut former de cette manière coïncident.

Quand $N=p q$ est un produit de deux nombres premiers on peut encore préciser les résultats précédents : Soit $S_{2}(p q, \mathbb{Z})$ l'espace des formes modulaires cuspidales de poids 2 sur $J_{0}(p q)$ à coefficients dans $\mathbb{Z}$. On désigne par $S^{\text {new }}\left(\operatorname{resp} S^{\text {old }}\right.$ ) le sous $\mathbb{Z}$-module de $S_{2}(p q, \mathbb{Z})$ engendré par les formes nouvelles (resp anciennes). On obtient alors

Théorème 1.3. Soit l un nombre premier ne divisant pas $6 p(p+1) q(q-1)$ et divisant $\left|J_{0}(q)\left(\mathbb{F}_{p^{2}}\right)\right|$. Il existe alors une forme $f \in S^{\text {new }}$ et une forme $g \in S^{o l d}$ telle que $f$ soit congrue à $g$ modulo $l$. On a de plus la minoration

(4) $\log c_{J_{0}(p q)}$ new $\geq \max \left(\frac{q-1}{6} \log (p-1), \frac{p-1}{6} \log (q-1)\right)-O(\log p q)$.

Indiquons brièvement le plan de ce texte : Dans la section 2, nous donnons les préliminaires de géométrie algébrique qui nous servirons à calculer la constante de Hida d'un quotient optimal de la jacobienne $J$ d'une courbe de genre $g \geq 2$ définie et semi-stable sur $\mathbb{Q}$ en fonction des fibres de mauvaise réduction du modèle de Néron de $J$. Ces résultats sont donnés dans la section 3 . On prouve les théorème 1.1 et 1.2 dans la section 4 . Le théorème 1.3 est démontré dans la dernière partie en utilisant les résultats de Mazur [9] et de Jordan-Livné [6].

Remerciements Je tiens à remercier chaleureusement le raporteur de ce texte pour de multiples remarques pertinentes qui m'ont permis de corriger de nombreuses imprécisions. C'est aussi un plaisir de remercier les organisateurs des journées arithmétiques de Rome pour leur invitation et la qualité de l'organisation de cette conférence.

\section{Isogénies autoduales}

Soit $\mathcal{O}$ un anneau de valuation discrète de corps des fractions $K$ et de corps résiduel $k$ de caractéristique $p>0$. Soit $A_{K}$ une variété abélienne sur $K$. On note $A$ son modèle de Néron, $A^{0}$ la composante connexe de l'élément neutre, $A_{k}$ et $A_{k}^{0}$ les fibres spéciales de $A$ et $A^{0}$ et $\phi(A, k)$ le groupe des composantes de $A_{k}$. On suppose que $A_{K}$ a réduction semi-stable sur $\mathcal{O}$. On a donc une suite exacte pour la topologie fppf :

$$
0 \rightarrow T_{k} \longrightarrow A_{k}^{0} \longrightarrow A_{k}^{*} \rightarrow 0
$$

où $A_{k}^{*}$ est une variété abélienne sur $k$ et $T_{k}$ est un tore.

Soit $u_{K}$ une isogénie $A_{K} \longrightarrow B_{K}$ de noyau $G_{K}$. Soit $u$ son extension au modéle de Néron et $u^{0}$ son extension au modéle de Néron connexe. On a 
alors une suite exacte pour la topologie fppf :

$$
0 \rightarrow G \longrightarrow A^{0} \longrightarrow B^{0} \rightarrow 0
$$

où $G$ est un schéma en groupe quasi-fini et plat $\operatorname{sur} S=\operatorname{Spec}(\mathcal{O})$ de fibre générique $G_{K}$. On note $G^{f}$ la partie finie de $G$ (la complétion de $G$ le long de sa fibre spéciale), $G^{t}$ sa partie torique et $G_{K}^{f}\left(\operatorname{resp} G_{K}^{t}\right)$ la fibre générique de $G^{f}\left(\operatorname{resp} G^{t}\right)$.

Pour tout schéma noethérien $S$ et tout schéma en groupe commutatif $L$, fini et plat sur $S$ on note $L^{\vee}$ le dual de Cartier de $L$. On a donc un accouplement de dualité sur $L \times L^{\vee}$. Pour tout sous-schéma en groupe $L^{\prime}$ de $L$, on note $L^{\prime \perp}$ le sous-schéma en groupe de $L^{\vee}$ orthogonal à $L^{\prime}$.

Soit $A_{K}^{\vee}$ la variété abélienne duale de $A_{K}$. Soit

$$
\lambda: A_{K} \longrightarrow A_{K}^{\vee}
$$

une isogénie. En identifiant $\left(A_{K}^{\vee}\right)^{\vee} \grave{a} A_{K}$, on dispose d'une isogénie

$$
\lambda^{\vee}: A_{K} \longrightarrow A_{K}^{\vee} \text {. }
$$

On dit que $\lambda$ est autoduale si $\lambda=\lambda^{\vee}$.

Le but de cette partie est de montrer le résultat suivant qui nous sera utile dans la suite de ce texte :

Proposition 2.1. On suppose que $A_{K}$ est une variété abélienne sur $K$ à réduction purement torique. Soit $A_{K}^{\vee}$ la variété abélienne duale et

$$
\lambda: A_{K} \longrightarrow A_{K}^{\vee}
$$

une isogénie autoduale de noyau $H_{K}$. Alors $\left|\left(H_{K}\right)\right|$ est un carré et

$$
\left|\left(H_{K}^{f}\right)\right|=\sqrt{\left|\left(H_{K}\right)\right|} .
$$

Pour toute variété abélienne $B_{K}$ sur $K$, on note ${ }_{n} B_{K}$ le noyau de l'isogénie $n_{B_{K}}$ multiplication par $n$ sur $B_{K}$. On sait que l'on a la relation

$$
\left({ }_{n} B_{K}\right)^{\vee}={ }_{n} B_{K}^{\vee} \text {. }
$$

Lemme 2.2. Soit a la dimension de $A_{K}$. Pour tout entier $n$ on a :

$$
\left|\left({ }_{n} A_{K}^{f}\right)\right|=n^{a}
$$

Preuve. L'isogénie $n_{A_{K}}$ induit la multiplication par $n$ sur la composante neutre de la fibre spéciale. Comme la réduction de $A_{K}$ est purement torique, le noyau de la multiplcation par $n$ est de cardinal $n^{a}$. On obtient le lemme en utilisant la définition de ${ }_{n} A_{K}^{f}$.

Lemme 2.3. Soit $n$ un entier. La restriction $\grave{a}_{n} A_{K}^{f} \times{ }_{n} A_{K}^{\vee f}$ de l'accouplement de dualité $\operatorname{sur}_{n} A_{K} \times{ }_{n} A_{K}^{\vee}$ est triviale. 
Preuve. En passant aux composantes $l$-primaires, on se ramène au cas où $n=l^{r}$ pour un nombre premier $l$. On note $T_{l}\left(A_{K}\right)$ le module de Tate de $A_{K}$. On a une filtration

$$
0 \subset T_{l}\left(A_{K}\right)^{t} \subset T_{l}\left(A_{K}\right)^{f} \subset T_{l}\left(A_{K}\right)
$$

où

$$
T_{l}\left(A_{K}\right)^{f}=\lim _{\leftarrow} l^{k} A_{K}^{f}(\bar{K})
$$

et

$$
T_{l}\left(A_{K}\right)^{t}=T_{l}\left(A_{K}\right)^{f}
$$

car $A$ est à réduction purement torique (ce qui nous dispense d'en donner une définition). Le théorème d'orthogonalité de Grothendieck ([5] théorème 5-2) nous assure que la restriction à $T_{l}\left(A_{K}\right)^{t} \times T_{l}\left(A_{K}^{\vee}\right)^{f}$ de l'accouplement sur $T_{l}\left(A_{K}\right) \times T_{l}\left(A_{K}^{\vee}\right)$ est triviale. Le lemme s'obtient alors en remarquant que l'accouplement sur $l^{r} A_{K}^{f} \times{ }_{l^{r}} A_{K}^{\vee f}$ est induit par celui sur $T_{l}\left(A_{K}\right)^{t} \times$ $T_{l}\left(A_{K}^{\vee}\right)^{f}$.

Lemme 2.4. Soit $n$ un entier. On a un isomorphisme

$$
{ }_{n} A_{K}^{\vee f} \simeq\left({ }_{n} A_{K} /{ }_{n} A_{K}^{f}\right)^{\vee} .
$$

Preuve. On a deux suites exactes :

$$
0 \longrightarrow{ }_{n} A_{K}^{f} \longrightarrow{ }_{n} A_{K} \longrightarrow{ }_{n} A_{K} /{ }_{n} A_{K}^{f} \longrightarrow 0
$$

et

$$
0 \longrightarrow\left({ }_{n} A_{K} /{ }_{n} A_{K}^{f}\right)^{\vee} \longrightarrow\left({ }_{n} A_{K}\right)^{\vee} \longrightarrow\left({ }_{n} A_{K}^{f}\right)^{\vee} \longrightarrow 0 .
$$

La dualité parfaite $\operatorname{sur}{ }_{n} A_{K} \times{ }_{n} A_{K}^{\vee}$ est triviale sur

$$
{ }_{n} A_{K}^{f} \times\left({ }_{n} A_{K} /{ }_{n} A_{K}^{f}\right)^{\vee}
$$

et induit la dualité de Cartier $\operatorname{sur}{ }_{n} A_{K}^{f} \times\left({ }_{n} A_{K}^{f}\right)^{\vee}$. On a donc

$$
\left({ }_{n} A_{K} /{ }_{n} A_{K}^{f}\right)^{\vee}=\left({ }_{n} A_{K}^{f}\right)^{\perp} \text {. }
$$

Mais d'après le lemme 2.3, on a :

$$
{ }_{n} A_{K}^{\vee f} \subset\left({ }_{n} A_{K}^{f}\right)^{\perp},
$$

mais d'après le lemme 2.2 , pour une raison de cardinalité, on a

$$
{ }_{n} A_{K}^{\vee f}=\left({ }_{n} A_{K}^{f}\right)^{\perp} .
$$

Ceci termine la preuve du lemme 2.4.

Lemme 2.5. La restriction à $H_{K}^{f} \times H_{K}^{f}$ de l'accouplement de dualité sur $H_{K} \times H_{K}$ est triviale. 
Preuve. Soit $n$ le cardinal de $H_{K}$. Soit $\delta: A_{K}^{\vee} \longrightarrow A_{K}$ l'isogénie telle que $\delta \circ \lambda=n_{A_{K}}$. Soit $I_{K}$ son noyau. On a la relation :

$$
I_{K}=\lambda\left({ }_{n} A_{K}\right)
$$

et $\delta$ est une isogénie autoduale. On a donc deux suites exactes :

$$
0 \longrightarrow H_{K} \longrightarrow{ }_{n} A_{K} \longrightarrow I_{K} \longrightarrow 0
$$

et

$$
0 \longrightarrow I_{K} \longrightarrow{ }_{n} A_{K}^{\vee} \longrightarrow H_{K} \longrightarrow 0 .
$$

Dans cette dernière suite exacte $H_{K}$ est réalisé comme l'image par $\delta$ de ${ }_{n} A_{K}^{\vee}$. On peut donc appliquer le lemme 4.4.5 de [11] et on obtient les deux suites exactes suivantes :

$$
0 \longrightarrow H_{K}^{f} \longrightarrow{ }_{n} A_{K}^{f} \longrightarrow I_{K}^{f} \longrightarrow 0
$$

et

$$
0 \longrightarrow I_{K}^{f} \longrightarrow{ }_{n} A_{K}^{\vee f} \longrightarrow H_{K}^{f} \longrightarrow 0 .
$$

L'accouplement sur $H_{K}^{f} \times H_{K}^{f}$ est trivial car il se déduit de l'accouplement sur ${ }_{n} A_{K}^{f} \times{ }_{n} A_{K}^{\vee f}$ qui est trivial d'après le lemme 2.3.

Lemme 2.6. On a l'inégalité :

$$
\left|\left(H_{K}^{f}\right)^{2}\right| \leq\left|\left(H_{K}\right)\right| .
$$

Preuve. C'est une conséquence immédiate de la relation

$$
\left|\left(H_{K}^{f}\right)\right|\left|\left(\left(H_{K}^{f}\right)^{\perp}\right)\right|=\left|\left(H_{K}\right)\right|
$$

et du lemme 2.5 qui nous assure que $H_{K}^{f} \subset\left(H_{K}^{f}\right)^{\perp}$.

Lemme 2.7. On a une inclusion naturelle de $K$-schéma en groupe :

$$
\left(H_{K} / H_{K}^{f}\right)^{\vee} \subset H_{K}^{f} \text {. }
$$

Preuve. On a un diagramme commutatif à lignes exactes :

$$
\begin{aligned}
& 0 \longrightarrow \begin{array}{c}
H_{K}^{f} \\
\downarrow
\end{array} \quad \begin{array}{c}
H_{K} \\
\downarrow
\end{array} \quad \begin{array}{c}
H_{K} / H_{K}^{f} \\
\downarrow
\end{array} \longrightarrow 0 \\
& 0 \longrightarrow{ }_{n} A_{K}^{f} \longrightarrow{ }_{n} A_{K} \longrightarrow{ }_{n} A_{K} /{ }_{n} A_{K}^{f} \longrightarrow 0 \text {. }
\end{aligned}
$$

On en déduit après passage au dual et en utilisant le lemme 2.4 le diagramme commutatif à ligne exactes :

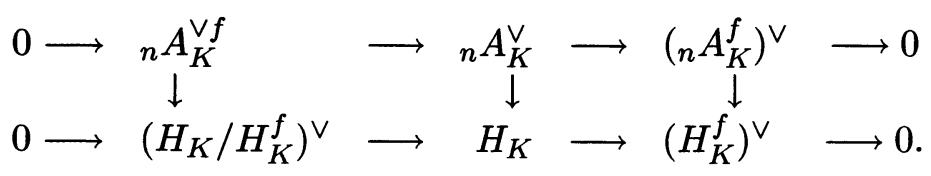


Le lemme s'obtient à partir de ce diagramme en utilisant l'équation (14) qui nous assure que l'image de ${ }_{n} A_{K}^{\vee f}$ dans $H_{K}$ est $H_{K}^{f}$.

Preuve de la proposition 2.1. Le lemme 2.7 prouve que

$$
\left|\left(H_{K} / H_{K}^{f}\right)\right| \leq\left|\left(H_{K}^{f}\right)\right| .
$$

La proposition 2.1 est donc conséquence du lemme 2.6.

\section{Constante de Hida et monodromie}

Soit $X$ une surface de Riemann et $J$ sa jacobienne. Rappelons que l'on a définie pour tout quotient optimal $A$ de $J$ la constante de Hida $c_{A}$ de $A$ [17]. Soit $\zeta: J \longrightarrow A$ le morphisme de variété abélienne donnant la structure de quotient. Soit $\zeta^{\vee}: A^{\vee} \longrightarrow J$ le morphisme dual. On dispose alors d'une isogénie autoduale $\zeta \zeta^{\vee}: A^{\vee} \longrightarrow A$ de noyau $H$ avec

$$
c_{A}=\sqrt{|H|} \text {. }
$$

Notons que la constante de Hida dépend en général du morphisme $\zeta$. La notation $c_{A}$ présuppose donc que $A$ est donné avec sa structure de quotient optimal. Nous pensons que cet abus de notation ne devrait pas poser de problème au lecteur. Dans les applications cette structure sera en fait unique par les théorèmes de multiplicité 1.

Soit maintenant $V$ une variété abélienne sur $\mathbb{Q}$ de dimension $r$. On suppose que $V$ a réduction semi-stable sur $\mathbb{Q}$. Soit $l$ une place de mauvaise réduction de $V$. On note $T(V, l)$ la partie torique de la composante neutre de la réduction du modèle de Néron de $V$ en $l, H(V, l)$ le groupe des caractères de $T(V, l)$ et $\phi(V, l)$ le groupe des composantes. On rappelle ([5, Théorème 10.4]) que l'on a un accouplement de monodromie canonique non dégénéré :

$$
u_{V}: H(V, l) \times H\left(V^{\vee}, l\right) \longrightarrow \mathbb{Z}
$$

et une suite exacte induite

$$
0 \longrightarrow H\left(V^{\vee}, l\right) \longrightarrow \operatorname{Hom}_{\mathbb{Z}}(H(V, l), \mathbb{Z}) \longrightarrow \phi(V, l) \longrightarrow 0 .
$$

Soit $t$ la dimension de $T(V, l)$. On note encore $u_{V}$ l'accouplement induit sur $\wedge^{t} H(V, l) \times \wedge^{t} H\left(V^{\vee}, l\right)$. On en déduit le lemme suivant qui nous sera utile dans la suite de ce papier.

Lemme 3.1. Soient $x$ et $y$ des générateurs de $\wedge^{t} H(V, l)$ et $\wedge^{t} H\left(V^{\vee}, l\right)$. On a alors

$$
\left|u_{V}(x, y)\right|=|\phi(V, l)| .
$$

On suppose maintenant que $X, J$ et $A$ sont définis sur $\mathbb{Q}$ que $J$ et $A$ sont à réduction purement torique en une place $l$. Soit $a$ la dimension de $A$. On dispose des morphismes induit

$$
\zeta^{*}: H(A, l) \longrightarrow H(J, l)
$$


et

$$
\zeta^{\vee *}: H(J, l) \longrightarrow H\left(A^{\vee}, l\right)
$$

On pose alors :

$$
\Theta(A, l)=\zeta^{*} H(A, l) \otimes_{\mathbb{Z}} \mathbb{Q} \cap H(J, l) .
$$

Proposition 3.2. On a $c_{A}=\left|\left(\operatorname{coker}\left(\zeta^{\vee *} \zeta^{*}\right)\right)\right|$

Preuve. Soient $\mathcal{A}$ et $\mathcal{A}^{\vee}$ les modèles de Néron de $A$ et $A^{\vee}$. Soient $\mathcal{A}^{0}$ et $\mathcal{A}^{\vee 0}$ les composantes neutres de $\mathcal{A}$ et $\mathcal{A}^{\vee}$. L'isogénie $\lambda=\zeta \zeta^{\vee}$ se prolonge au modèles de Néron connexe et on a une suite exacte pour la topologie fppf :

$$
0 \longrightarrow H \longrightarrow \mathcal{A}^{\vee 0} \longrightarrow \mathcal{A}^{0} \longrightarrow 0 .
$$

Le noyau de l'isogénie induite sur les tores est alors $H^{f} \otimes \mathbb{Z} / l \mathbb{Z}$. On en déduit alors que

$$
\left|\left(\operatorname{coker}\left(\zeta^{\vee *} \zeta^{*}\right)\right)\right|=\left|H_{f}\right|
$$

La proposition résulte alors de l'équation (17) et de la proposition 2.1.

On note $u_{J}$ l'accouplement de monodromie induit sur

$$
\bigwedge^{a} \Theta(A, l) \times \bigwedge^{a} \Theta(A, l)
$$

Soit $e$ un générateur du $\mathbb{Z}$-module libre de rang $1, \bigwedge^{a} \Theta(A, l)$ et $\tau=u_{J}(e, e)$. Proposition 3.3. On a la relation :

$$
c_{A}|(\phi(A, l))|=\left[\Theta(A, l): \zeta^{*} H(A, l)\right]^{2} \tau .
$$

Preuve. Soit $x$ et $y$ des générateurs de $\bigwedge^{a} H(A, l)$ et de $\bigwedge^{a} H\left(A^{\vee}, l\right)$. D'après le lemme 3.1 , on a

$$
\left|u_{A}(x, y)\right|=|(\phi(A, l))| .
$$

D'après la proposition $3.2, x$ étant fixé, on peut choisir $y=\zeta^{\vee *} \zeta^{*} x$. On en déduit que

$$
\left|u_{A}\left(x, \zeta^{\vee *} \zeta^{*} x\right)\right|=c_{A}\left|u_{A}(x, y)\right|=c_{A}|(\phi(A, l))| .
$$

D'autre part, on a la relation

$$
\left|u_{A}\left(x, \zeta^{\vee *} \zeta^{*} x\right)\right|=\left|u_{J}\left(\zeta^{*} x, \zeta^{*} x\right)\right|=\left[\Theta(A, l): \zeta^{*} H(A, l)\right]^{2} \tau .
$$

(Remarquer que $\tau$ est positif) Ceci termine la preuve de la proposition.

Lemme 3.4. Soit $\zeta_{*}: \phi(J, l) \longrightarrow \phi(A, l)$ le morphisme induit par $\zeta$. On a la relation:

$$
\left[\Theta(A, l): \zeta^{*} H(A, l)\right]=\left|\left(\operatorname{coker} \zeta_{*}\right)\right|
$$


Preuve. Comme $\left[\Theta(A, l): \zeta^{*} H(A, l)\right]$ est le cardinal de la partie de torsion du conoyau de l'application $\zeta^{*}: H(A, l) \longrightarrow H(J, l)$, on peut aussi le calculer comme le cardinal du conoyau de l'application

$$
\operatorname{Hom}\left(\zeta^{*}, \mathbb{Z}\right): \operatorname{Hom}_{\mathbb{Z}}(H(J, l), \mathbb{Z}) \longrightarrow \operatorname{Hom}_{\mathbb{Z}}(H(A, l), \mathbb{Z}) .
$$

On obtient alors le lemme quand on applique le lemme du serpent au diagramme commutatif à lignes exactes

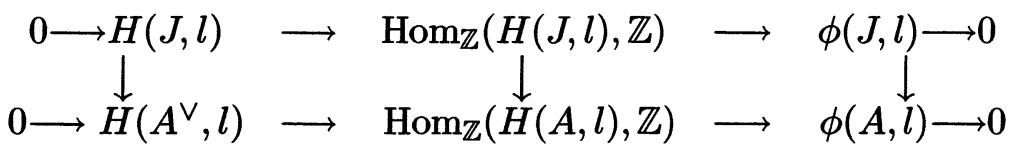

quand on a remarqué que la flèche verticale de gauche est surjective puisque l'application $\zeta^{*}$ induit une immersion fermée sur les modèles de Néron connexe de $A^{\vee}$ et $J$. (Noter que si $l>2$, on a même une immersion fermée au niveau des modèles de Néron d'après [1, Théorème $4, p .186]$.

Le rapporteur m'a fait remarquer qu'il est difficile de donner une référence pour la commutativité du carré de droite dans le diagramme commutatif précédent. Nous espérons que le lecteur courageux écrira une preuve de ce fait.

\section{Quotients de jacobiennes de courbes de Shimura}

Soit $N$ un entier sans facteurs carrés. Soit $N=N^{+} N^{-}$une décomposition de $N$ avec $N^{-}$un produit d'un nombre pair de nombres premiers. Soit $X_{N^{+}, N^{-}}$la courbe de Shimura définie dans l'introduction et $J_{0}\left(N^{+}, N^{-}\right)$ sa jacobienne. Soit $p$ et $q$ deux facteurs premiers de $N^{-}$. On pose alors

$$
J_{1}=J_{0}\left(N^{+}, N^{-}\right), \quad J_{2}=J_{0}\left(N^{+} p q, \frac{N^{-}}{p q}\right) \quad \text { et } \quad J_{3}=J_{0}\left(N^{+} p, \frac{N^{-}}{p q}\right) .
$$

On a alors la proposition suivante ([16, proposition 1]), obtenue par Ribet [13] quand $N^{-}=p q$ (ie $\left.J_{2}=J_{0}(N)\right)$ et qui se généralise grâce aux travaux de Buzzard [3] :

Proposition 4.1. On a une suite exacte canonique :

$$
0 \rightarrow H\left(J_{1}, p\right) \stackrel{i}{\longrightarrow} H\left(J_{2}, q\right) \rightarrow H\left(J_{3}, q\right) \times H\left(J_{3}, q\right) \rightarrow 0 .
$$

Cette suite est compatible à l'action des opérateurs de Hecke $T_{l},(l, N)=1$, sur chacun des termes de la suite. De plus l'application $i$ est compatible avec l'accouplement de monodromie : pour tout $(x, y) \in H\left(J_{1}, p\right)$ on a

$$
u_{J_{1}}(x, y)=u_{J_{2}}(i(x), i(y)) \text {. }
$$

Soit $A$ un quotient optimal de la partie nouvelle $J_{0}^{n e w}(N)$ de $J_{0}(N)$. Par la correspondance de Jacquet-Langlands, il existe des quotients optimaux $A_{1}, A_{2}$ de $J_{1}$ et $J_{2}$ qui sont isogène à $A$. Pour $i=1$ ou $i=2$, on note $\zeta_{i}$ l'application de $J_{i}$ vers $A_{i}$ qui s'en déduit. 
Lemme 4.2. On a la relation

$$
i\left(\Theta\left(A_{1}, p\right)\right)=\Theta\left(A_{2}, q\right)
$$

Pour tout nombre premier $l$ divisant $N$, le modèle de Néron de $J_{0}^{\text {new }}(N)$ est à réduction purement torique. Si $N=l$ cela résulte de la description de Mazur de la jacobienne $J_{0}(l)$ en $l$. Sinon soit $l^{\prime}$ tel que $l l^{\prime}$ divise $N$. Par Jacquet-Langlands, on sait que $J_{0}^{\text {new }}(N)$ est isogène à la partie nouvelle $J_{l l^{\prime}, \frac{N}{l^{\prime}}}^{n e w}$ de $J_{l l^{\prime}, \frac{N}{l^{\prime}}}$. Le théorème de Cerednik-Drinfeld assure que $J_{l l^{\prime}, \frac{N}{l^{\prime}}}$ est à réduction purement torique en $l$. On obtient le résultat car la propriété d'être à réduction purement torique est stable par isogénies et par passages au quotient. On déduit de cela que $A_{1}$ et $A_{2}$ sont à réduction purement torique en $p$ et $q$ et que $H\left(A_{1}, p\right)$ et $H\left(A_{2}, q\right)$ sont des $\mathbb{Z}$-modules libres de rang $a=\operatorname{dim}(A)$. Soit $\left(f_{1}, f_{2}, \ldots, f_{r}\right)$ les formes nouvelles de poids 2 sur $A$. Pour tout entier $n$, on écrit $T_{n} f_{i}=a_{n}\left(f_{i}\right) f_{i}$. On a alors

$$
\Theta\left(A_{1}, p\right)=H\left(J_{1}, p\right) \cap \oplus_{i=1}^{r}\left(H\left(J_{1}, p\right) \otimes \mathbb{R}\right)_{f_{i}}
$$

où

$\left(H\left(J_{1}, p\right) \otimes \mathbb{R}\right)_{f_{i}}=\left\{x \in H\left(J_{1}, p\right) \otimes \mathbb{R} \mid \forall n \in \mathbb{N},(N, n)=1, T_{n} . x=a_{n}\left(f_{i}\right) \cdot x\right\}$.

On finit la preuve du lemme en utilisant une description analogue pour $\Theta\left(A_{2}, q\right)$ et le fait que $i$ a un conoyau sans torsion et est compatible à l'action des opérateurs de Hecke pour $n$ premier à $N$.

Pour tout nombre premier $l$ divisant $N$, on note

$$
\zeta_{i, l *}: \phi\left(J_{i}, l\right) \longrightarrow \phi\left(A_{i}, l\right)
$$

le morphisme induit par $\zeta_{i}$. On obtient alors en utilisant les équations $(21,24,25,26)$ la relation

$$
\frac{c_{A_{1}}\left|\phi\left(A_{1}, p\right)\right|}{\left|\operatorname{coker} \zeta_{1, p *}\right|^{2}}=\frac{c_{A_{2}}\left|\phi\left(A_{2}, q\right)\right|}{\left.\left.\mid \operatorname{coker} \zeta_{2, q *}\right)\right)^{2} \mid} .
$$

Ceci termine la preuve du théorème 1.1.

\section{La courbe modulaire $J_{0}(p q)$}

Quand $N=p q$, le théorème 1.2 prend la forme très simple suivante : Soit

$$
\zeta: J_{0}(p q) \rightarrow J_{0}(p q)^{n e w}
$$

le morphisme donnant la structure de quotient optimal de $J_{0}(p q)^{\text {new }}$. On a la relation

$$
c_{0}=c_{J_{0}(N)^{n e w}}=\frac{\left|\phi\left(J_{0}(1, p q), p\right)\right|\left|\phi\left(J_{0}(p q)^{n e w}, q\right)\right|}{\left|\operatorname{Im}\left(\zeta_{q *}\right)\right|^{2}}
$$

Pour expliciter cette relation nous utiliserons le fait que $\phi\left(J_{0}(p q)^{n e w}, q\right)$ et $c_{0}$ sont des entiers, la description de $\phi\left(J_{0}(p q), q\right)$ dû à Mazur (qui permet de majorer le terme $\mid \operatorname{Im}\left(\left.\zeta_{q} *\right|^{2}\right.$ ) [9] (voir aussi [12]) et la description 
de $\phi\left(J_{0}(1, p q), p\right)$ dû à Jordan et Livné [6]. Décrivons brièvement leurs résultats. Pour simplifier les énoncés nous ne donnerons que les parties premières à 6 de ces groupes. Pour des nombres rationnels $a$ et $b$, on écrit $a \simeq b$ si leurs parties première à 6 coïncident. Mazur ([9, p. 174-175]) prouve que l'on a

$$
\left|\phi\left(J_{0}(p q), q\right)\right| \simeq(q-1)(p+1)
$$

et

$$
\left|\phi\left(J_{0}(p q), q\right)\right|=O(p q) .
$$

On en déduit que $\left|\operatorname{Im}\left(\zeta_{q *}\right)\right|=O(p q)$.

Soit $\mathbb{B}$ l'algèbre de quaternion indéfinie de discriminant $p q$ et $\widehat{\mathbb{B}}$ l'algèbre de quaternion définie de discriminant $q$. Soit $B(p)$ la matrice de Brandt de degré $p$ pour $\widehat{\mathbb{B}}$ relative aux classe d'idéaux de $\widehat{\mathbb{B}}[4]$. C'est une matrice carré d'ordre $h$ où

$$
h=\frac{1}{4}\left(1-\left(\frac{-4}{q}\right)\right)+\frac{1}{3}\left(1-\left(\frac{-3}{q}\right)\right)+\frac{q-1}{12} .
$$

(avec les conventions $\left(\frac{-4}{2}\right)=0=\left(\frac{-3}{3}\right)$.) Soit $g(n)$ le genre de $X_{0}(n)$, on a alors $h=g(q)+1$. On peut écrire le polynôme caractéristique $P(x)$ de $B$ sous la forme

$$
P(x)=(x-p-1) \prod_{i=2}^{h}\left(x-\lambda_{i}\right)
$$

où les $\lambda_{i}$ sont les valeurs propres de l'opérateur de Hecke agissant sur l'espace des formes modulaires cuspidales de poids 2 sur $X_{0}(q)$ [4]. Comme une base de cette espace est donnée par les new-forms, on dispose de la majoration

$$
\left|\lambda_{i}\right| \leq 2 \sqrt{p}
$$

On a alors le résultat suivant dû à Jordan et Livné [6]

$$
\left|\phi\left(J_{0}(1, p q), p\right)\right| \simeq \frac{p+1}{q-1}\left|\prod_{i=2}^{h}(p+1)^{2}-\lambda_{i}^{2}\right|
$$

On déduit des deux dernières équations (plus précisément de la forme donnée dans [6] de l'équation (34)) et de la valeur de $h$ donnée par (32) que l'on a l'estimation suivante :

$$
\log \left(\left|\phi\left(J_{0}(1, p q), p\right)\right|\right) \geq \frac{q-1}{6} \log (p-1)-O(\log (p q)) .
$$

Par ailleurs un calcul simple prouve que l'on a l'égalité

$$
\left|\prod_{i=2}^{h}(p+1)^{2}-\lambda_{i}^{2}\right|=\left|J_{0}(q)\left(\mathbb{F}_{p^{2}}\right)\right| \text {. }
$$


On obtient alors la preuve de la première partie du théorème 1.3 en utilisant les équations (29) (30) et (34) et le théorème 5-2 de [17] qui nous assure qu'un nombre premier $l$ ne divisant pas $2 p q$ mais divisant la constante de Hida $c_{J_{0}(p q)^{\text {new }}}$ à la propriété de l'énoncé du théorème 1.3. La deuxième partie est une simple conséquence des équations (29), (31) et (35) éventuellement en inversant le rôle de $p$ et $q$.

\section{Bibliographie}

[1] S. Bosch, W. Lutkebohmert, M. Raynaud, Néron models. Springer-Verlag New York, 1990.

[2] M. Bertolini, H. DARmon, Heegner points on Mumford-Tate curves. Invent. Math 126 (1996),no. 3, 413-456.

[3] K. Buzzard, Integral models of certain Shimura curves. Duke. Math. Journ. 87 (1997), $591-612$.

[4] B. Gross, Heights and the Special values of L-series. Canadian Math. Soc. Conf. Proc. 7 (1997), 115-187.

[5] A. GRothendIECK, Groupe de Monodromie en Géométrie Algébrique. SGA 7 I, Lecture notes in Math. 288, Springer Verlag, 1972.

[6] B. Jordan, R. Livne, On the Néron Model of Jacobian of Shimura Curves. Compositio Math. 60 (1986), 227-236

[7] H. HIDA, Congruences for cusps forms and special values of their zeta functions. Invent. Math 63 (1981), 225-261.

[8] H. HIDA, On congruence divisors of cusp forms as factors of the special values of their zeta functions. Invent. Math 64 (1981), 225-261.

[9] B. MAzur, Modular Curves and the Eisenstein Ideal. Pub. Math. IHES 47 (1977), 33-186.

[10] B. MAZUR, Rational isogenies of prime degree. Invent. Math 44 (1978), 129-162.

[11] M. RaYnaUd, Hauteurs et isogénies. Astérisque 127 (1985), 199-234.

[12] M. RAYNAUD, Jacobienne des courbes modulaires. Astérisque 196-197 (1991), 9-25.

[13] K. RIBET, On modular representations of $r m$ Gal $(\overline{\mathbb{Q}} / \mathbb{Q})$ arising from modular forms. Invent. Math. 100 (1990), 431-471.

[14] K. RiBET, Congruence relation between modular forms. Proc. Int. Congr. Math. (1983), 503-514.

[15] K. RIBET, Mod p Hecke operators and congruences between modular forms. Invent. Math. (1983), 193-205.

[16] K. RiBet, S. TAKAhASHI, Parametrisation of elliptic curves by Shimura curves and by classical modular curves. Proc. Natl. Acad. Sci. USA 94 (1997), 11110-11114.

[17] E. Ullmo, Hauteur de Faltings de quotients de $J_{0}(N)$, discriminants d'algèbres de Hecke et congruences entre formes modulaires. Amer. J. of Math. 122 (2000), 83-115.

[18] M.-F. Vignéras, Arithmétique des algèbres de quaternions. Lect. Notes. Maths 800, Springer-Verlag New-York, 1980.

Emmanuel Ullmo

Princeton University

Departement of Maths. Fine Hall

08540 Princeton NJ

USA

E-mail : eullmo@math.Princeton.edu 